\title{
Dissecting our impact factor
}

\author{
The papers we published in 2008 and 2009 received on average 29.9 citations each in 2010. However, \\ nearly $30 \%$ of them were cited more than 30 times, contributing to roughly two-thirds of the impact factor.
}

Single-number metrics such as the impact factor are useful, yet often simplistic. Although very relevant to librarians, publishers and scientists alike - especially when facing decisions constrained by shrinking budgets or career prospects - a journal's impact factor is only an averaged account of the citations to its content. To provide you readers with a nuanced view of the impact ${ }^{1,2}$ of our authors' papers, in this Editorial we delve into the 2010 impact factor of Nature Materials.

The average of a normally distributed variable is the value that occurs most frequently (in statistical terms, the mode). But this is of course not true in the skewed distribution of a journal's received citations, which are known to approximately follow a Pareto probability distribution - also known in bibliometrics as Bradford's law ${ }^{3}$. The Pareto distribution is a power-law function characterized by a long tail - in this context a long tail of highly cited papers - and it is commonly used to explain income distributions or stock-market returns, for example. The fact that the mode of a longtailed distribution strongly differs from the average is apparent in the histogram of citations for Nature Materials shown by the black bars in Fig. 1. Whereas the impact factor - the average - is approximately 30 , the most frequent number of citations per paper is roughly 7 .

Cumulative data (red curves in Fig. 1) paint yet a more revealing picture: $16 \%$ of the papers we published in 2008-2009 contributed to half of the citations received in 2010 (and thus to half of the impact factor), and half of the papers we published in the same period acquired $17 \%$ of the citations. Similarly, roughly $30 \%$ of the papers we published contributed to twothirds of the impact factor.

Review articles typically collect more citations than research articles, and therefore journals that publish reviews often lift their impact factor. At Nature Materials, out of the 263 research papers that we published in 2008-2009, 19 were reviews, which contributed to $17 \%$ of the citations. Citations to news pieces and editorial content which only supplement the impact factor's numerator - summed $5.4 \%$ of the total in 2010. (Note that in Fig. 1 we show only citations to papers, and thus the curve for

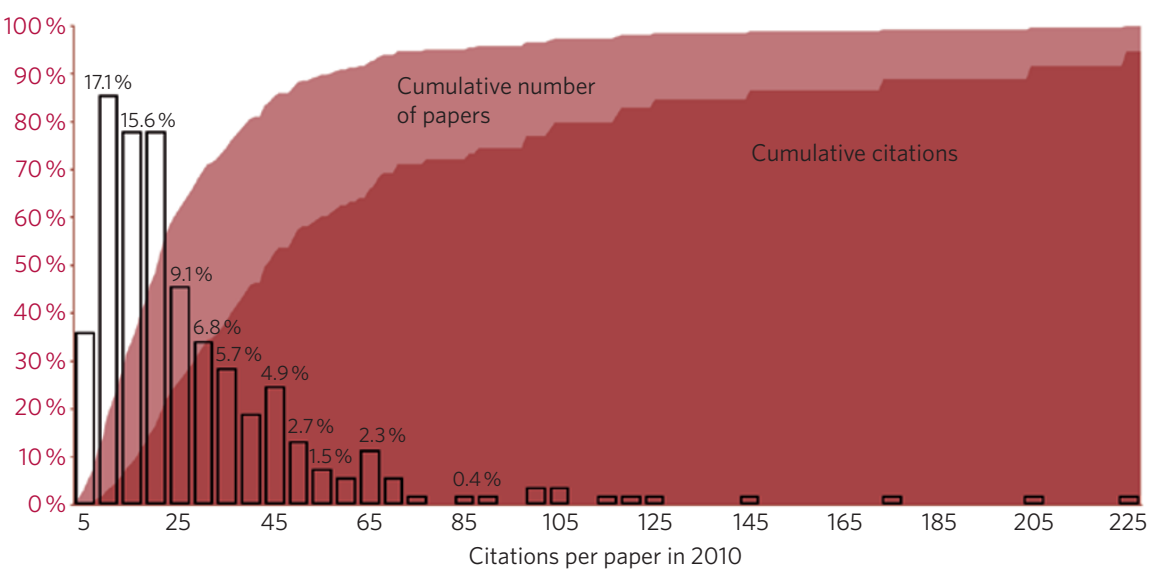

Figure 1 | Distribution of citations, received in 2010, to papers published in Nature Materials in 2008 and 2009. The bars indicate the percentage of papers (numbers in black) per intervals of citations per paper (0-4, 5-9 and so on). The cumulative number of papers (light red) and citations (dark red) are shown as percentages (vertical axis; numbers in red) of their totals. Data source: Thomson Reuters Web of Science ${ }^{4}$. Citation data from Web of Science slightly differs from that of Journal Citation Reports - used to calculate the impact factor - as a result of differences in indexing and citation windows ${ }^{5}$.

the cumulative citations misses 5.4 points to reach $100 \%$.)

Of course, different fields exhibit different citation patterns. For multidisciplinary journals, therefore, excessive focus on capturing the highly cited works in the long tail of the distribution could eventually tarnish the readers' perceptions of a journal's stance. On one hand, the bare number of short-term citations a paper receives is in some instances a mediocre proxy for the quality of the work. On the other hand, a journal's inflow of potentially highly cited papers could easily be volatile, as such papers tend to be few in number and often belong to selected trendy topics. At Nature Materials we are pleased by the variety of fields covered in the 6 reviews and 17 research articles constituting our long tail - here arbitrarily defined by the papers published in 2008-2009 with each receiving 60 or more citations in 2010 - in subject areas such as photovoltaics, multiferroics, spintronics, graphene, metamaterials and biomaterials, to mention a few.

Obviously, journals with a broad multidisciplinary audience are likely to publish highly cited papers that strengthen the long tail. However, in our opinion the long tail is only one trait - and not the main trait - of the complexion of Nature Materials. Actually, the shape of the cumulative curves and the histogram of citations, in particular the weight of the 'middle zone' - say, between one and two times the impact factor - may provide further clues about the nature of a journal. In fact, we are glad of the tall and paunchy shape of the histogram of citations in Fig. 1. In other words, we published a large number of papers that have been cited at a slower pace (the tall bars), as well as papers that have already collected a moderate amount of citations (the 'middle zone'). Topics include, for example, crumpling, granular materials, phase-change materials, hydrogels and glassy dynamics.

Citation metrics significantly affect how journals are assessed and perceived, and we hope that the overtones of the impact-factor song here discussed provide a more subtle sketch of the character of Nature Materials, as well as of the impact of our authors' papers according to citation numbers. And irrespective of post-publication metrics and potential citations, we will continue to do our best at publishing your most scientifically outstanding work in all areas of materials science.

References

1. Nature Mater. 10, 477 (2011)

2. Nature Mater. 3, 495 (2004).

3. Brookes, B. C. Nature 224, 953 (1969).

4. http://go.nature.com/Xf8YK6

5. http://go.nature.com/aznrxP 